\title{
Flow chamber for living cell detection by SPR
}

\author{
Fuyin Dou, Xinglong Yu, Lili Ding, Fangfang Liu \\ Dept. of Precision Instruments, Tsinghua University, Beijing 100084, P. R. China, \\ Corresponding author's e-mail: jyxyxl@mail.tsinghua.edu.cn
}

\begin{abstract}
:
The detection of molecular interactions on living cells using SPR is attracting more and more attention. This paper presents a flow chamber design for maintaining suitable physiological conditions of living cells during detection. It includes a microfluidic flow system providing precise injection of culture media or reagent and a themoregulator assuring suitable temperature. Experimental results demonstrate that the flow chamber is reliable for detecting living cells.
\end{abstract}

Key words: living cell detection, molecular interactions, flow chamber, SPR

\section{Introduction}

It's not only a challenge but a precondition to research molecular interactions on living cells for further cellomic research. This new research field needs new research method to support it. Surface plasmon resonance(SPR) sensing is a sensitive, label-free and real-time optical detection method. The combination of SPR with cell-based biosensing can provide not only the specificity, affinity and kinetic constant of molecular interactions on living cells but also the information of cell adhesion, spreading and migration. Nowadays, it has been a new research trend, and the number of publications is steadily growing[1].

There are two crucial premise conditions for detecting living cells based on SPR sensing: one is cell culture on SPR chip, and the other is microenvironment maintenance. We made use of the technique of microfludic and PID temperature control to design and implement the flow chamber. Experimental results show that it can meet the requirement for detecting living cells.

\section{Design and fabrication}

The structure of the flow chamber is shown in Fig.1. The bottom of the device is a copper plate which works as the base of the device and the cooling fin of the Thermoelectric cooling (TEC) device. Two side PMMA plates are respectively fixed on the end of the copper plate for holding the Kretschmann prism, and the prism is stick on the top of the TEC by thermo conductive grease. The cell culture chamber made of PDMS is pressed on the gold film by the top PMMA plate fixed on the side PMMA plates. A thermistor is casted in the culture chamber, and the temperature inside the culture chamber is regulated by a PID close loop controller. The microfluidic flow system could precisely inject various reagents through a multiway valve and high precision pumps. All the components were designed with the software of Solidworks. The cell culture chamber was fabricated using PDMS replica molding, and the master mold and PMMA plates were fabricated by laser ablation. The photo of the device is shown in Fig. 2 .

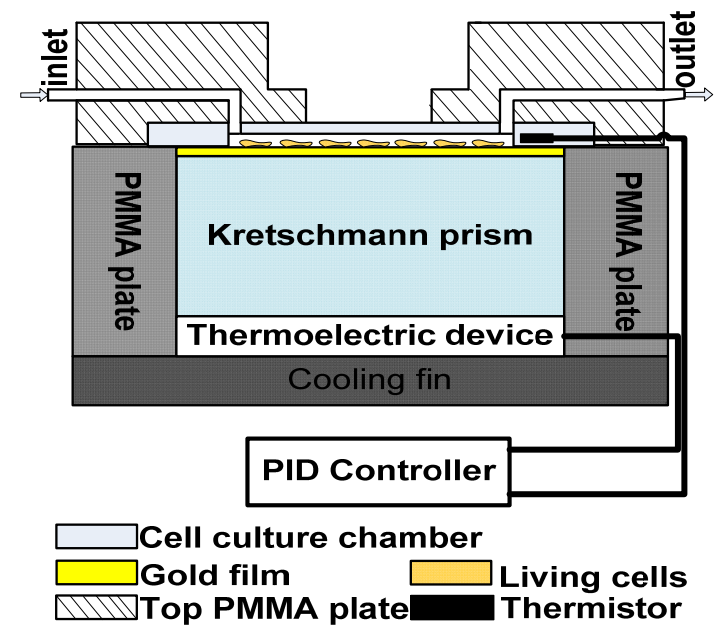

Fig. 1. Schematic of the structure of the flow chamber.

\section{Experiment and results}

3.1 Temperature stability: Culture media was continuously injected into the culture chamber by a peristaltic pump at the rate of $20 \mu \mathrm{l} / \mathrm{min}$ and the controlled temperature was set to $37^{\circ} \mathrm{C}$. After the temperature was stable, fluctuation of the temperature was recorded for more than 3 hours. The result is shown in Fig.3. The result 


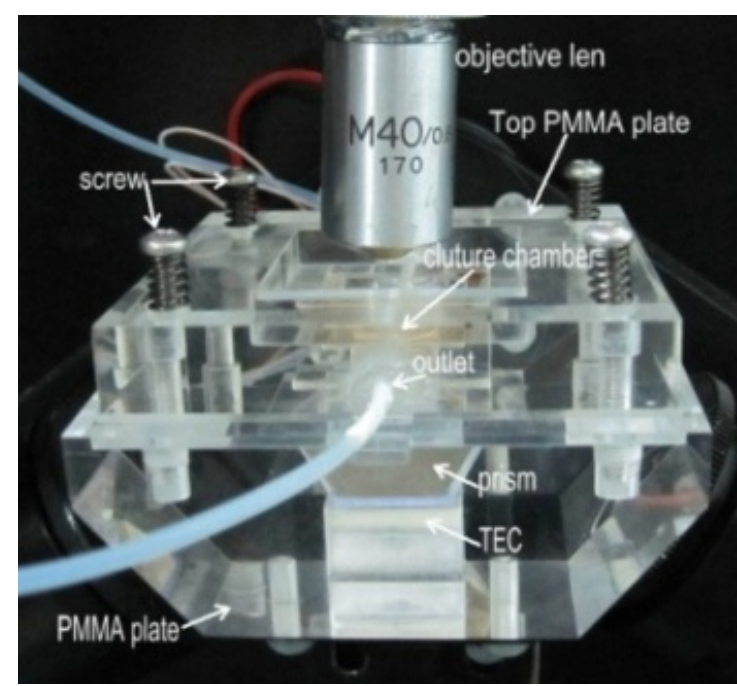

Fig. 2. Photo of the flow chamber.

shows that the fluctuation of the temperature is less than $\pm 0.2^{\circ} \mathrm{C}$ and the temperature could meet the need of living cells. The precision can be improved if the structures further optimized.

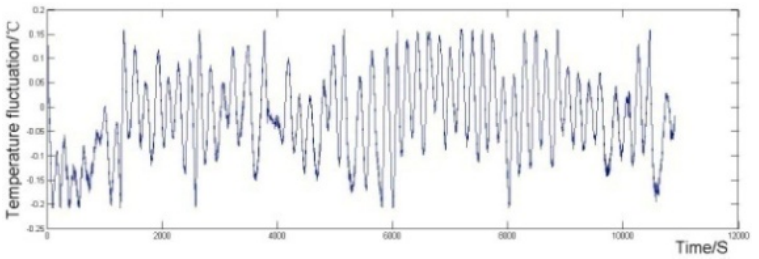

Fig. 3. The fluctuation of the temperature.

3.2 Cell culture: When the temperature was stable, BGC-823 cells were seed in the cell culture chamber. Then culture media was ontinuously injected into the culture chamber by a peristaltic pump at the rate of $5 \mu \mathrm{l} / \mathrm{min}$. A microscope was used to monitor the attachment and spreading of the living cells.Fig. 4 shows the pictures of $5 \mathrm{~h}, 15 \mathrm{~h}$ and $30 \mathrm{~h}$ after the cells was seed. The result indicates that the cells can be successfully cultured in the chamber.

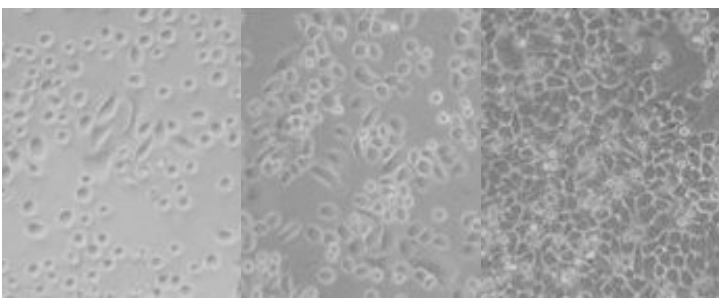

Fig. 4. Cell culture in the chamber.

3.2 Cell culture: When the temperature was stable, BGC-823 cells were seed in the cell culture chamber. Then culture media was ontinuously injected into the culture chamber by a peristaltic pump at the rate of $5 \mu \mathrm{l} / \mathrm{min}$. A microscope was used to monitor the attachment and spreading of the living cells.Fig. 4 shows the pictures of $5 \mathrm{~h}, 15 \mathrm{~h}$ and $30 \mathrm{~h}$ after the cells was seed. The result indicates that the cells can be successfully cultured in the chamber.

3.3 Ligand/cell interaction: The device was mounted on a SPR system which was further described elsewhere [2], and the BGC-823 cells was cultured for $15 \mathrm{~h}$ according to the above method. Anti-EGFR at different concentrations prepared with cell culture media was used to respectively stimulate the cells for $25 \mathrm{~min}$. Cell culture media was injected into the chamber as the baseline before stimulation. The results are shown in Fig.5.It is obvious that the cells have different response to anti-EGFR on different concentrations.

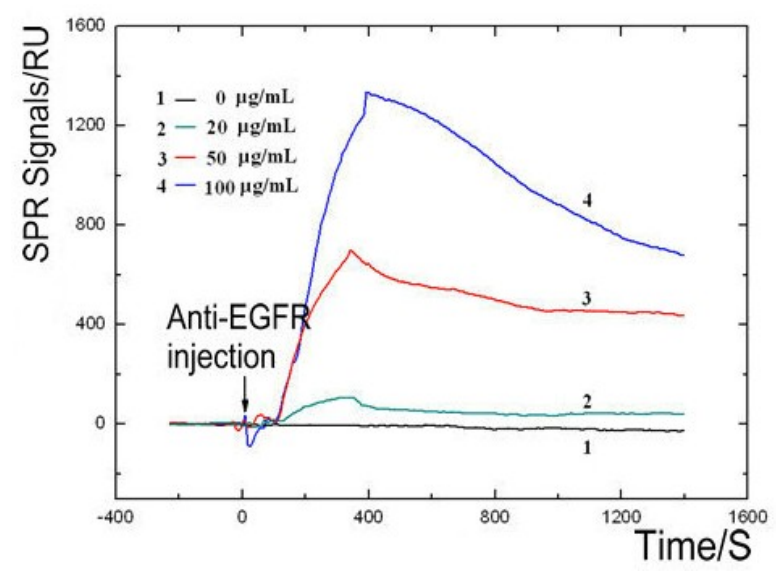

Fig. 4. SPR response signals.

\section{Conclusion}

The experimental results indicate that the flow chamber provides a stable microenvironment for living cells and it's reliable for continuously and controllably detecting the living cells.

\section{Acknowledgements}

This work was supported by the National Natural Science Foundation of China (Grant No. 30970757 and30727001)

\section{References}

[1] R. Robelek, Surface plasmon resonance sensors in cell biology: basics and application, Bioanal $\operatorname{Rev} 1$, 57-72(2009); doi: 10.1007/s12566-0090005-y

[2] X. L. Yu, X. Ding, F. F. Liu, Y. Deng, A novel surface plasmon resonance imaging interferometry for protein array detection, Sensors and Actuators B 130,52-58 (2008); DOI: 10.1016/j.snb.2007.07.106 